\title{
La familia rifeña $y$ el espacio
}

Abdeluahab Hammouti

\section{Resumen}

Las transformaciones acaecidas en el Rif Oriental desde la irrupción colonial española hasta la actualidad, han generado una nueva dinámica de las estructuras sociales, económicas e ideológicas. En este sentido, los pilares de la sociedad rifeña tradicional, preurbana y solidariamente organizada en torno a la familia extensa, han ido desapareciendo gradualmente, para dar paso a una novedosa configuración donde los aspectos tradicionales quedan incluidos en los nuevos parámetros impuestos por las sociedades urbanas, que en nuestro caso, contempla como principal agente dinamizador, la espectacular transformación urbanistica de la ciudad de Nador.

\section{La institución familiar}

En el estado actual de nuestras investigaciones ${ }^{1}$ podemos afirmar la total inexistencia de las instituciones clásicas relacionadas con

(1) Este artículo es la traducción española de un extracto de la tesis presentada en la Facultad de Letras y Ciencias Humanas de la Universidad de Fez, en el Departamento de Sociología Urbana, bajo el título: Las transformaciones urbanas en el Rif Oriental: Nador como modelo, redactada en árabe. 
Abdeluahab Hammouti

la kábila (tribu). La nueva estructura de la sociedad marroqur ha alcanzado este estadio después de haber conocido un régimen estatal rigido. Es decir, un estado moderno cuyas instituciones difieren de las antiguas.

Es cierto que las instituciones clásicas (económicas, sociales, políticas, etc.) organizaban la sociedad rifeña ordenándola segmentariamente en kábilas, jemaas, d' chares y, por último, los individuos. Las instituciones nuevas, por el contrario, organizan al individuo respecto a la sociedad. En definitiva, las primeras instituciones se interesan por el grupo y las segundas por el individuo.

A pesar de la negación de las kábilas, los rasgos de los kabileños permanecen en lo que podemos llamar tribalismo que es, según nuestra visión, un conjunto de ideas o de opiniones que producía la kábila para organizarse, imponer su identidad, identificarse y para reproducirse.

La alusión a lo kabileño, hoy en día, se ha convertido, claramente, en una ideología política apologética cuyo fin es defender unas actitudes de partido o de clases sociales. Por ejemplo, hay discursos políticos que insisten sobre la "thwiza"2, sobre el trabajo colectivo, o sobre la noción de jemmaa (colectividad o comunidad), particularmente cuando se trata de las elecciones comunales o parlamentarias. Muchos entonces efectúan pocas diferencias entre la noción de jemaa de kábila (comunidad tribal) y la noción de comunidad actual. Igualmente muchos mezclan las nociones del kaid de kábila y del kaid en su nuevo aspecto en la actual estructura de la sociedad marroquí.

Creemos que la situación a que está sometida la sociedad marroquí rompe totalmente con la etapa anterior, es decir, con la etapa preurbana. La continuación de lo kabileño (tribalismo) sólo interesa a algunas esferas muy determinadas, como la esfera política (elecciones, partidos, etc.). Esto se aclara cuando observamos algunos comportamientos de determinadas clases de individuos, ancianos especialmente. Estos últimos, aun saturados de razonamientos antiguos pertenecientes a la jemaa o kábila, encuentran grandes dificultades para adaptarse a la nueva situación. Por lo que hoy en día podemos afirmar la disfunción del razonamiento kabileño, ya que se ha convertido en una idcología política. Podemos añadir, también, que a esta tendencia política calificada de frágil,

(2) La thwiza es el trabajo colectivo que solía marcar la actividad cotidiana u ccasional de los rifeños en el pasado kabileño. 
no le queda ninguna base material concreta para subsistir.

Teniendo en cuenta esto, podemos concluir que la zona del Rif Oriental no se limita a unos simples conjuntos de kábilas sino que es una "provincia" según el vocabulario del estado moderno y las instituciones que la articulan y organizan no son diferentes a las instituciones de otras provincias del país.

Las instituciones del nuevo estado encuentran a través de las ciudades ${ }^{3}$ sus itinerarios hacia cada familia y cada individuo, particularmente para controlar su comportamiento, forjar sus ideas, formar su lengua, seguir su estado de salud mental o corporal, vigilar sus tendencias polfticas y sentimentales. Todo ello, para integrarlo y dominarlo. El fin de estas instituciones actuales es producir y reproducir la sociedad y lo social continuamente.

Sin duda, la zona del Rif Oriental ha conocido un desarrollo social que ha instaurado una ruptura con el pasado kabileño o con lo tradicional. El desarrollo mencionado es consecuencia del fenómeno urbanístico al que está relacionado con las instituciones que organizan la zona. Así, las instituciones educativas, de enseñanza pública, las sanitarias, cooperativas agrícolas o inmobiliarias, las sociedades financieras o bancarias, etc., no pertenecen a un pasado kabileño de la zona, caracterizado por un preurbanismo, sino que pertenecen a un presente urbanístico muy significativo.

Acabamos de exponer la imagen estructural de la nueva sociedad que ha elegido la ciudad o lo urbano como espacio específico para sus múltiples y nuevas instituciones.

En este apartado tratamos de analizar una institución social fundamental: la familia rifeña, y ello por muchas razones. En primer lugar, porque esta institución no es totalmente nueva sino antigua. Ciertamente la kábila ha conocido dicha institución, pero nadie contesta ahora la sumisión de la familia rifeña a los mecanismos de la renovación y del cambio. Sus funciones, por ejemplo, han sido transformadas hasta el punto de ser contradictorias con las del pasado kabileño. En segundo término, porque la familia como estructura social representa un espacio

(3) La ciudad no se reduce a un espacio topográfico o geográfico sobre cl cual se dispersan los individuos, sino, según nuestra visión, se conforma, en primer lugar, en un espacio social institucional. Un espacio controlado, dirigido por medios represivos, que determinan una autoridad sistematizada sin precedentes. 
Abdeluahab Hammouti

social especifico en el cual podemos leer los mecanismos del poder, de la producción y reproducción, de la orientación, de la represión, etc. Y por último, porque la familia como producción social será el espacio elemental que nos ayude a entender los procesos de la integración.

\section{De la familia extensa a la familia unitaria}

El acontecimiento más grandioso que ha marcado la historia de la institución familiar en el Rif Oriental ha sido la transformación que ha afectado a su estructura. Antes era una familia extensa, ahora lo es unitaria o nuclear. Esta transformación fue el resultado de los cambios de las estructuras socioeconomicas.

La sociedad urbana, al igual que la economia urbana, ha generado unas nuevas relaciones, culturales y políticas que han determinado la naturaleza de la familia rifeña moderna. Esta condición es igual y afecta a toda la sociedad marroquí en vías de una urbanización constante. La aparición de las ciudades y el desarrollo de las estructuras urbanas modernas imponen, efectivamente, unas formas nuevas de relaciones sociales. En este contexto podemos comprender por qué la familia rifeña actual no difiere de las demás familias marroquíes. Según nuestra hipótesis es una institución sometida al cuadro de las relaciones urbanas actuales, a los factores múltiples de índole económico-social, al proceso de la modernización, etc. Por tanto, la familia rifeña es un producto socioeconómico a través del cual se propagan los poderes, las ideologías, y los diferentes comportamientos: morales, religiosos o de valores.

\section{La familia rifeña tradicional}

Antiguamente, la familia rifeña era extensa ya que la formaban varios elementos: los abuelos, los hijos casados y los sobrinos. Estos elementos estaban ordenados jerárquicamente de arriba a abajo: abuelo, hijo casado, sobrino. La rclación que los ordenaba es la del poder expresado por la autoridad del tutor, del padre, del abuelo o del primogénito (los "mayores" de la familia). Estas relaciones del poder detentaban su legalidad por medio de las relaciones parentales y, en general, por medio de las relaciones sociales kabileñas existentes. El jefe de la familia rifeña - generalmente se trataba de un hombre - era su representante simbólico o personal en los consejos de las jemaas. Era el responsable de 
todos sus elementos y el destino de éstos dependía de él. Por ejemplo, el jefe de familia intervenía en el matrimonio de los hijos o hijas y asímismo en su vida futura, dirigfa los trabajos del campo, transmitra las ideas, las informaciones y las sugerencias de la jemaa o del consejo de la kábila a su hogar y viceversa. En suma, el jefe de familia, se preocupaba de lo exterior al hogar, organizando las relaciones mutuas entre su familia y la jemaa.

En cuanto a la mujer (madre, abuela o hija), su tarea estaba muy ligada a la casa. Por ejemplo, se preocupaba de la cocina, del agua, de los animales domésticos, etc. Pero no estaba subordinada al hombre, no estaba sometida a la explotación sexual o económica, a pesar de la división del trabajo observado entre ella y el hombre.

Habra un cierto intercambio de las ideas que incluían una democracia en las decisiones que interesaban al hogar, los niños, las normas del campo y de la agricultura o del zoco, etc.

La mujer rifeña no demostraba una carencia de personalidad o una mala reputación en el dominio de las relaciones kabilefas. Al contrario, era un miembro que gozaba de todos los derechos materiales e inmateriales. A veces, por lo que respecta al honor de su descendencia, sus hijos e hijas podían afiliarse nominalmente a ella.

Además de extensa, la familia rifeña estaba unida. Las relaciones parentales o consanguíneas solidificaban esa unión tal como lo hace la circulación de los bienes económicos sometidos al modo de producción tribal. La propiedad de la tierra, con respecto a ese modo de producción, era colectiva. Los productos eran destinados al consumo colectivo. La idea de comercializarlos estaba poco admitida. Por encima, intervienen en este juego, los sistemas morales y culturales para reforzar esa unión y perpetuar el nombre de la kábila, guardar su honor y su linaje.

Por otra parte, la familia rifeña vivía bajo el mismo techo: la vivienda rifeña era una construcción de forma cuadrada o rectangular, muy amplia. Sus ventanas y puertas se abrian sobre espacioso patio. La función de este patio es interpretada de la manera siguiente: el rifeño sale de su cuarto directamente al patio para encontrar el cielo o Dios. Nosotros interpretamos que el patio era un lugar para ejercer la vida común o unida. Es decir, un lugar para los encuentros diarios u ocasionales de los elementos de la familia extensa. La vivienda extensa implica lógicamente, la amplitud de la familia. 
La familia rifeña tradicional era extensa, unida y solidaria. Este estado lo reflejan los espacios como las viviendas, las jemaas, las confederaciones kabileñas, etc. Pero la colonización cambió esta estructura familiar radicalmente. La familia rifeña fue el blanco primordial del colonialismo español que propago muchos medios para desestructurarla y romper las relaciones parentales existentes entre sus muchos elementos. Esta tarea estaba incluida en las estrategias de la dominación y de la producción de una nueva sociedad que convenía más a la condición colonial. Insistimos sobre el hecho de que la colonización española en el Rif Oriental no veŕa su estabilidad, su enraizamientos, sino sólo a través de las nuevas instituciones económicas, sociales, urbanas, lingüísticas, militares, administrativas, etc. Era una necesidad primordial el que estas instituciones reemplazaran a las instituciones kabileñas tradicionales porque la kábila, básicamente, procede de una lógica de violencia y de enfrentamiento directo, es decir, que las negociaciones frías o políticas no cuentan en su estrategia. Es el ejemplo de las guerras del Rif de 1921 a 1926.

Entre los medios que emplearon los españoles para reestructurar la familia rifeña y en consecuencia las jemaas y las kábilas, se observa, entre otros el de la monetarización. Se sabe, por ejemplo, que los españoles crearon varias empresas y medios de trabajo (como la construcción del puerto de Melilla, los lavaderos de mineral en el norte de Nador, las minas del Rif, etc.). Todo ello, para transformar a los rifeños en obreros remunerados y sometidos a la concurrencia, entre ellos, por los puestos de trabajo y la búsqueda de un buen sueldo. Esta nueva condición ha cambiado, en el plano económico, muchos conceptos como el de trabajo colectivo (thwiza) y ha introducido otros nuevos como la repartición del tiempo y la búsqueda de beneficios. Así, los españoles sembraron las primeras semillas de una sociedad que tiende ahora hacia el capitalismo.

En el plano social surgió la explotación de los individuos. El hombre $y$, particularmente, la mujer rifeña han dejado la familia extensa en busca de trabajo en los centros urbanizados. Aquí no ignoramos la aparición de una capa de beneficiarios de la condición colonial.

En efecto, es difícil tratar el tema de la desestructuración de la familia rifeña en el periodo colonial porque faltan los datos y los documentos resultan inaccesibles por muchas razones. Respecto a este tema se plantea un obstáculo frente a la escritura objetiva de la historia social o de la sociología de la zona del Rif Oriental. 


\section{La familia rifeña moderna}

La familia rifeña actual es una familia nuclear constituida por los siguientes elementos: padre, madre e hijos. En esto se diferencia de la familia rifeña tradicional, pudiendo confirmarse una ruptura entre ambos modelos. En efecto, la familia rifeña moderna es una institución nueva que se mueve en un espacio institucional nuevo que ha roto con la sociedad kabileña preurbana. Se observa en este contexto que las instituciones marroqufes actuales obedecen a una lógica homogénea por lo que resulta insólita la discusión sobre las instituciones kabileñas que conservan aún sus funcionamientos tradicionales.

Las familias rifeñas actuales tienden a concentrarse en el espacio urbano, en Nador o en los centros periféricos. La emigración ha acentuado esta atracción. Más de 12.000 personas se instalaron en Nador entre 1960 y 1970 . Teóricamente no se puede entender la emigración como un simple desplazamiento en el espacio geográfico sino como un desplazamiento de primera categoría, es decir, como una transición de una estructura a otra: de las kábilas a las ciudades y centros urbanos. Este desplazamiento hacia las ciudades es temporal y su persistencia está limitada porque depende de la ausencia de las infraestructuras urbanas en el mundo rural como las escuelas, transportes, aguas, electricidad, carreteras, etc.

Generalmente la familia rifeña actual, ya sea en su forma o en su función, se encuentra regularizada sistemáticamente por el mecanismo urbanístico.

Por lo que concierne a la forma, la familia rifeña está reducida a unos elementos espaciales limitados. Padre, madre e hijos conviven en unas construcciones que les convienen: los apartamentos. Estos últimos pueden ser diferentes respecto al número de habitaciones pero no lo son en cuanto a la ausencia de los patios. Lo que afirma, que las construcciones de Nador, ordinariamente de forma cúbica, están destinadas a un solo fin que es el de dormir. Por eso se observa la ausencia de los patios en los apartamentos y por otra parte, la presencia de las calles, las plazas públicas, los diferentes centros sociales (cafés, clubs, centros deportivos, salas de fiestas, salas de conferencias, etc.) inexistentes en el ámbito rural antiguo.

Por lo que se refiere a la función, la familia rifeña se ha convertido en una institución que reproduce las relaciones de producción 
actualmente existentes. Es semejante a una empresa que crea individuos y los dota de sus ideas (función biológica y función ideológica o educativa). A veces, la familia puede limitarse a la primera función (biologica) y las otras instituciones como la escuela, la calle, el idioma, etc., se preocupan del aspecto funcional ideológico, educativo o autoritario.

Aquf el poder del padre puede ser neutralizado porque el sistema del poder personal del jefe de familia ha perdido su estado tradicional y ha pasado de los individuos a las instituciones modernas. Es decir, hay una transición del poder personal directo de las familias rifeñas clásicas al poder simbólico, impersonal, de las actuales. 